\title{
Influence de différentes structures de commande sur le compromis temps/vibration - Application à une machine UGV
}

\author{
Frédéric Colas ${ }^{\mathrm{a}}$, Jean-Yves Dieulot et Pierre-Jean Barre \\ Équipe de Recherche CEMODYNE, Laboratoire d'Électrotechnique et d'Électronique de Puissance de Lille (L2EP), ENSAM, \\ 8 avenue Louis XIV, 59046 Lille Cedex, France
}

Reçu le 15 mars 2007, accepté le 6 juillet 2007

\begin{abstract}
Résumé - L'augmentation des performances d'une machine de positionnement passe obligatoirement par une augmentation de ses sollicitations. Il est alors nécessaire d'améliorer soit sa structure mécanique, soit sa structure de commande. Dans ce dernier cas, le choix d'une structure de commande adaptée peut améliorer les performances et réduire les vibrations venant des modes propres d'un système. Cet article propose une étude comparative de l'influence de différentes structures de commandes sur le compromis temps/vibration. Cette analyse a été réalisée sur un robot cartésien. La liste des commandes retenues est loin d'être exhaustive, cependant celles qui font l'objet de cette étude sont directement utilisables sur les systèmes actuels. Après avoir présenté le système sur lequel repose cette étude, chaque architecture commande retenue est analysée et les résultats expérimentaux obtenus pour chacune sont présentés.
\end{abstract}

Mots clés : Structures de commande / temps de cycle / vibration / système flexible

\begin{abstract}
Control algorithm influence on the compromise movement time/vibration - Application to a high speed machine. While improving the dynamical performance of a flexible motion control system, additional stresses are generated which induce undesirable vibrations. The design of adequate control algorithms makes it possible to enhance the overall behaviour of the machine while limiting its deformation modes vibrations. This paper presents a comparative study of the influence of different control algorithms on the trade-off between the cycle time and the amplitude of the residual vibrations, which have been applied to a Cartesian robot. While this selection of control algorithms is not exhaustive, these can all easily be implemented in current industrial systems. Each selected control algorithm is analysed along with experimental validations.
\end{abstract}

Key words: Control algorithm / movement time / residual vibration / flexible system

\section{Introduction}

Les robots manipulateurs sont utilisés dans de nombreuses applications industrielles et notamment dans l'industrie plastique où ils servent généralement à la décharge de presses à injection plastique. L'objectif est évidemment dans la réduction des temps de cycle et des coûts de production. Cette réduction passe nécessairement par la diminution des inerties de toute nature et par l'augmentation des sollicitations en termes d'efforts imposés aux structures. Ces dispositions conjointes

\footnotetext{
a Auteur pour correspondance :

frederic.colas@centraliens-lille.org
}

conduisent à un comportement dynamique qui doit être pris en compte dans la phase de conception : la réduction des masses fait prévaloir le caractère élastique des liaisons et des structures, amenant ainsi une situation de modes oscillatoires dans la bande des fréquences d'utilisation de ces systèmes. La vélocité et la précision dynamique s'en trouvent considérablement affectées, ce qui va à l'encontre des objectifs visés. Il en découle, pour la pièce manipulée, une perte sensible de précision de son positionnement dynamique, ce qui, de toute évidence, devient préjudiciable par rapport aux objectifs de réduction globale des coûts. Il existe des savoirs scientifiques en matière de stratégies d'asservissements, mais il convient de les faire évoluer en 


\section{Nomenclature}

\begin{tabular}{|c|c|}
\hline$a$ & Amortissement du modèle masse-ressort (N.s.m ${ }^{-1}$ ) \\
\hline$A_{\max }:$ & Valeur de l'accélération maximum $\left({\mathrm{m} . \mathrm{s}^{-2}}^{2}\right)$ \\
\hline$f:$ & Coefficient de frottements visqueux (N.s.m ${ }^{-1}$ ) \\
\hline$J_{\max }:$ & Valeur de jerk maximum $\left(\mathrm{m} \cdot \mathrm{s}^{-3}\right)$ \\
\hline$k:$ & Raideur du modèle masse-ressort $\left(\mathrm{N} \cdot \mathrm{m}^{-1}\right)$ \\
\hline$k_{1}$ : & Gain de vitesse de la commande par retour accélérométrique (N.s.m $\left.{ }^{-1}\right)$ \\
\hline$k_{2}:$ & Gain de position de la commande par retour accélérométrique $\left(\mathrm{N} \cdot \mathrm{m}^{-1}\right)$ \\
\hline$K_{\mathrm{v}}:$ & Gain proportionnel de vitesse de la commande classique (A.s.rad ${ }^{-1}$ ) \\
\hline$K_{\mathrm{v}}:$ & Gain de vitesse de la commande par retour d'état (A.s.m $\left.{ }^{-1}\right)$ \\
\hline$K_{\mathrm{p}}:$ & Gain proportionnel de position de la commande classique $\left(\mathrm{s}^{-1}\right)$ \\
\hline$K_{\mathrm{p}}:$ & Gain de position de la commande par retour d'état $\left(\mathrm{A} \cdot \mathrm{m}^{-1}\right)$ \\
\hline$K_{\mathrm{i}}:$ & Gain intégral de la commande par retour d'état (A.(m.s $\left.\left.{ }^{-1}\right)\right)$ \\
\hline$M_{1}:$ & Masse modale $1 \mathrm{du}$ modèle masse-ressort $(\mathrm{kg})$ \\
\hline$M_{2}:$ & Masse modale 2 du modèle masse-ressort $(\mathrm{kg})$ \\
\hline$M_{\text {éq }}:$ & Masse totale en mouvement $(\mathrm{kg})$ \\
\hline$\tau_{\mathrm{i}}:$ & Constante de temps intégrale de vitesse de la commande classique (s) \\
\hline$U:$ & Effort de poussée provenant du moteur $(\mathrm{N})$ \\
\hline$x:$ & Position moteur $(\mathrm{m})$ \\
\hline$x_{\text {réf }}:$ & Référence de position moteur (m) \\
\hline$x_{1}:$ & Position de la masse $m_{1}(\mathrm{~m})$ \\
\hline$x_{2}:$ & Position de la masse $m_{2}(\mathrm{~m})$ \\
\hline$\dot{y}:$ & Dérivée de $y$ \\
\hline
\end{tabular}

prenant en compte le contexte particulier dans lequel nous nous trouvons.

Cette étude se propose de réaliser une analyse comparative de l'influence de différentes architectures de commande sur les performances en temps de cycle et en comportement vibratoire d'un robot cartésien. L'objectif est de montrer que les algorithmes de commande communément rencontrés ne sont pas les plus performants pour notre cas d'étude. Les différentes architectures de commande retenues sont les suivantes : commande par boucles en cascades avec référence en bang-bang d'accélération ou en bang-bang de jerk, commande par retour d'état basé sur un modèle rigide, commande par boucles en cascades avec filtrage de la mesure vitesse par filtre de Kalman, commande par retour accélérométrique. Cette liste n'est pas exhaustive [1] mais les commandes retenues ont l'avantage d'être implantables dans les systèmes actuels.

\section{Système étudié}

\subsection{Architecture globale}

Le robot sur lequel repose l'ensemble de cette étude est un robot cartésien 3 axes. Il est principalement utilisé en industrie pour venir chercher des pièces moulées dans une presse à injection plastique et pour les placer ensuite dans une zone de stockage ou sur un dispositif de convoyage. Ce robot constitué de trois axes orthogonaux $(X, Y$ et $Z$ ) ainsi que son architecture globale est présenté figure 1 . La motorisation est réalisée par des moteurs synchrones
Brushless à entraînement direct. Les transformations de mouvement de l'axe $X$ et $Z$ sont assurées par un système pignon-crémaillère. Par contre, le mouvement effectué suivant l'axe $Y$ est réalisé par un système poulie-courroie. La seule mesure disponible pour contrôler ce système est une mesure de position de l'arbre moteur par l'intermédiaire d'un resolver placé sur celui-ci. Dans toute la suite de cet article, la position moteur correspond à la mesure de position de l'arbre moteur multipliée par le rayon du pignon placé en sortie d'arbre moteur.

Comme nous l'avons précisé dans l'introduction, les sollicitations dynamiques au niveau de chaque axe excitent les premiers modes vibratoires de la structure et par conséquent détériorent la précision dynamique du système. Cette précision ne constitue pas une caractéristique fondamentale pour ce type de manipulateur. Par contre, les oscillations visibles de l'organe terminal (portant la charge) lors des phases d'arrêt, peuvent se révéler inacceptables. En des termes plus commerciaux, la confiance de l'opérateur sera fortement conditionnée par la qualité visuelle du comportement de la machine. L'amélioration de ce comportement peut passer par une meilleure architecture de commande. Il est cependant nécessaire d'établir un cahier des charges précis qui constituera notre référence de réglage. Pour chacune des architectures de commande testées dans cette étude, le robot implanté dans notre laboratoire a été réglé de façon à optimiser le temps de cycle en se fixant un seuil de dépassement au niveau de la position moteur. Le cahier des charges est défini par les points suivants :

- le temps de cycle : il est déterminé lorsque l'on atteint la position de consigne $-0,5 \mathrm{~mm}$, 

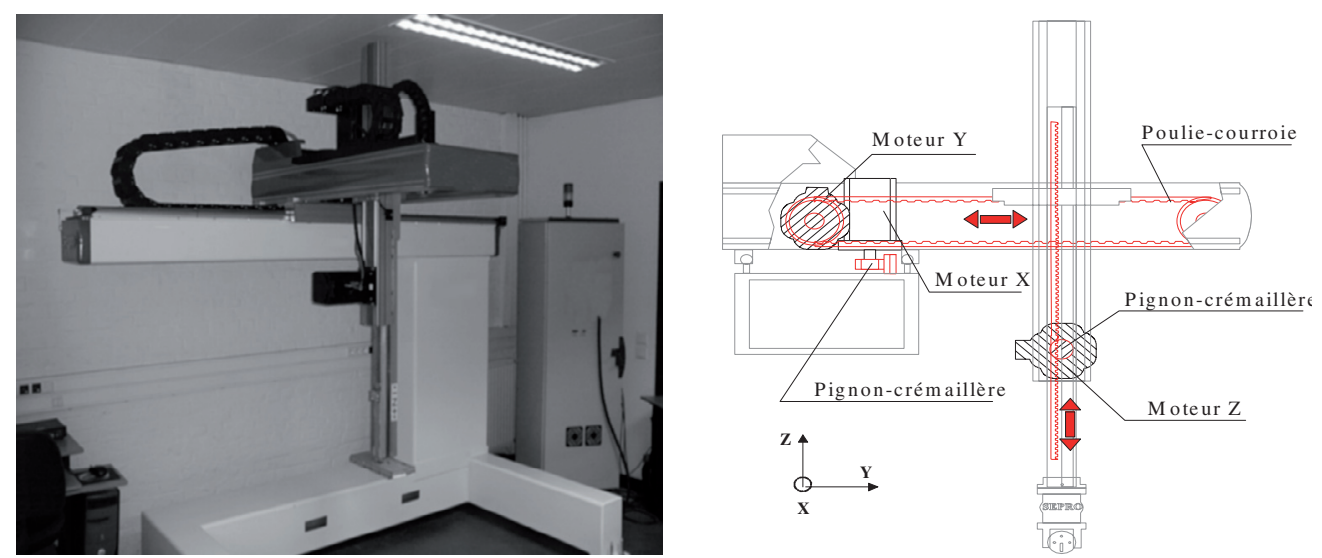

Fig. 1. Système étudié (robot cartésien 3 axes).

- les oscillations au niveau moteur : elles ne doivent pas dépasser le «tube d'acceptation » de $-0,5$ à $+0,2 \mathrm{~mm}$ de la consigne finale à atteindre,

- les oscillations de la masse finale : il n'existe pas de valeurs prédéfinies, en réalité, c'est une appréciation visuelle de spécialistes qui déterminent si le comportement est acceptable ou non. Elle sera définie par une valeur d'amortissement $(\zeta)$.

La figure 2 montre des mesures effectuées sur notre manipulateur pour un déplacement suivant la direction $Y$ de $200 \mathrm{~mm}$. On peut donc en déduire pour ce cas particulier un temps de cycle de 0,47 s et un amortissement des oscillations de la masse finale obtenu par interpolation exponentielle de 0,16 . Il est important de constater que la position de la charge (en bout de bras $Z$ ) est très oscillante et de ce fait difficile à commander.

\subsection{Causes des vibrations}

Les causes de vibration de ce robot manipulateur sont multiples. Cependant, il est possible d'en lister les principales :

- Il n'existe pas de mesure effective sur le véritable point à contrôler. Le bout du bras n'est en effet pas équipé de capteur et son contrôle se fait donc en boucle ouverte.

- Les signaux de position et vitesse moteur sont bruités du fait de la qualité du resolver, de leurs quantifications et du traitement numérique effectués sur ceuxci. La dérivée numérique du signal de position moteur pour obtenir la mesure vitesse introduit énormément de bruits.

- Le système est flexible et très peu amorti, le facteur d'amortissement est de l'ordre 0,1. Le comportement vibratoire est, de plus, très variable selon la position du bras. La fréquence de résonance varie, par exemple, du simple au double sur l'axe $Y$.

- La connaissance des paramètres de ce système est loin d'être précise. Les incertitudes paramétriques notamment sur le facteur d'amortissement des modes vibratoires sont élevées.

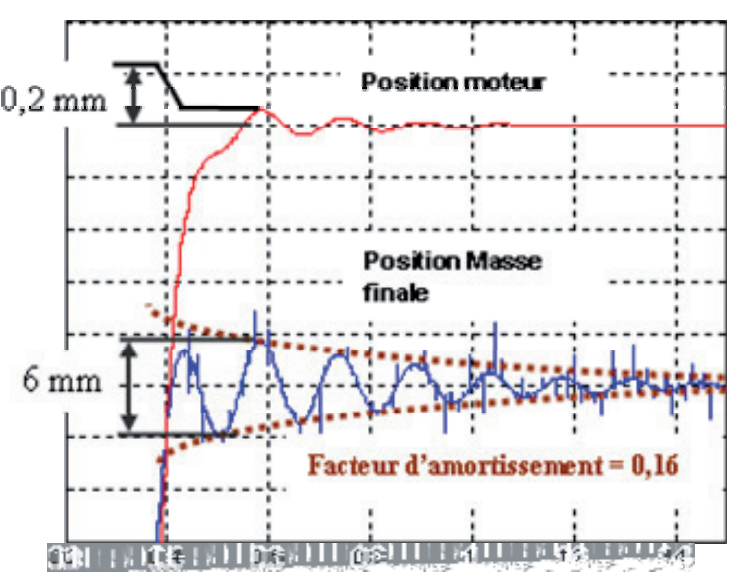

Fig. 2. Exemple de relevés effectués pour un déplacement en $Y$ de $200 \mathrm{~mm}$.

- Le rapport entre l'inertie de la charge en mouvement et l'inertie moteur est élevé et varie de 20 à 35 . Le comportement de l'arbre moteur est donc très oscillant [2].

\subsection{Expérimentations réalisées}

Toutes les expérimentations qui suivent ont été réalisées sur l'axe $X$ du manipulateur. Les caractéristiques de cet axe sont résumées dans la figure 3. La trajectoire qui nous sert de référence correspond à un déplacement arbitraire de $900 \mathrm{~mm}$ en bang-bang d'accélération à accélération et décélération maximales. Elle est présentée figure 3. En théorie, la durée totale de ce mouvement est de $0,95 \mathrm{~s}$ et la vitesse maximale atteinte de $1,9 \mathrm{~m} . \mathrm{s}^{-1}$.

Chacune des commandes a été implantée dans un environnement temps réel (dSPACE) qui pilote les variateurs de puissances du robot en courant. Les résultats expérimentaux sont tous résumés dans une figure type qui présente l'écart entre la référence de position de la figure 3 et l'évolution de la position moteur, 


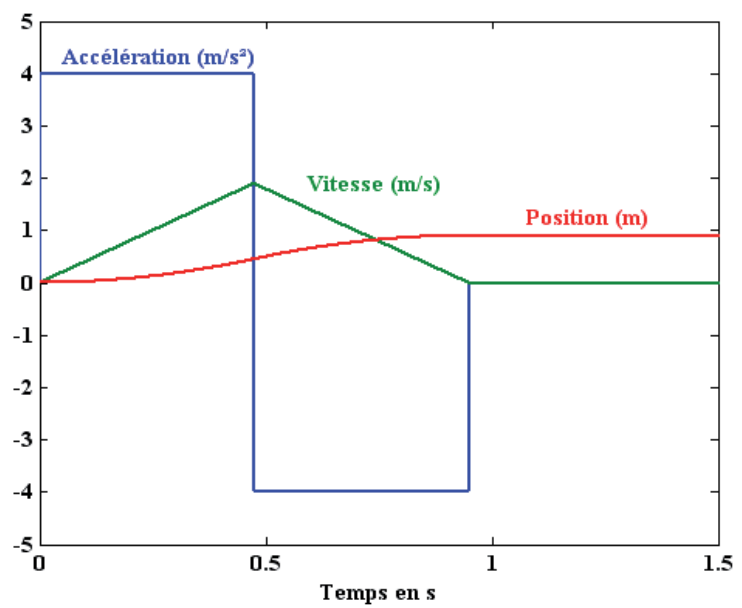

\begin{tabular}{|c|c|}
\hline Course max. & $1750 \mathrm{~mm}$ \\
\hline Accélération max. & $4 \mathrm{~m} / \mathrm{s}^{2}$ \\
\hline Vitesse max. & $4 \mathrm{~m} / \mathrm{s}$ \\
\hline Charge transportée & $12 \mathrm{~kg}$ \\
\hline Masse mobile & $205 \mathrm{~kg}$ \\
\hline
\end{tabular}

Fig. 3. Trajectoire de référence utilisée pour les différents types de commande et caractéristiques génériques de l'axe $X$.

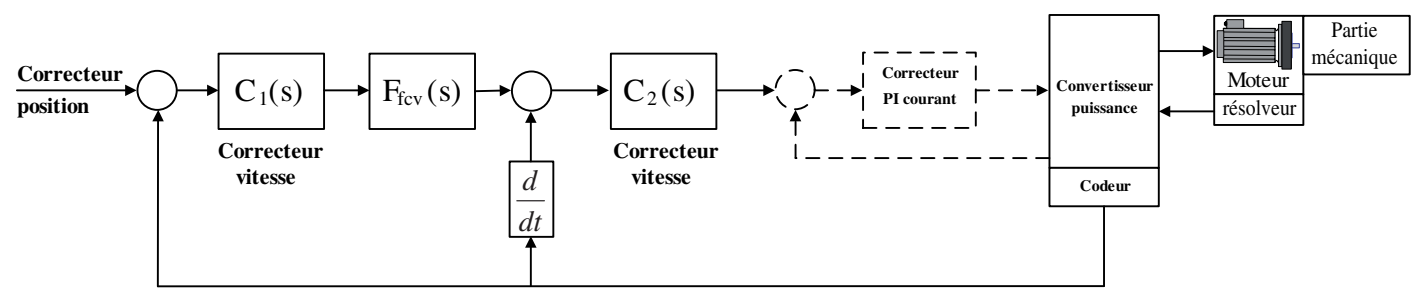

Fig. 4. Structure de la commande « classique».

l'évolution du courant moteur et une mesure provenant d'un capteur laser qui permet de mesurer les vibrations de la charge en fin de mouvement.

\section{Commande « classique $»$}

La commande dite «classique » correspond à celle utilisée industriellement [3]. Elle est fréquemment rencontrée dans la commande d'axe de machines outils, de robots cartésiens ou de machines à dynamiques élevées. Elle correspond à une structure en boucles en cascades (Fig. 4). La majeure partie des commandes numériques utilise cette structure de commande. Elle est composée de trois boucles fermées de dynamique croissante.

La boucle de courant (la plus rapide) est constituée d'un correcteur PI (Proportionnel-Intégral). Sa dynamique étant très élevée par rapport aux autres phénomènes physiques intervenant dans le système, elle sera toujours négligée et nous considérerons dans toute la suite de cette étude que le courant moteur est équivalent à sa consigne. Toutes les architectures de commande testées fournissent donc une consigne de courant de référence. Le variateur de puissance reste donc en charge de la régulation de courant. La boucle de vitesse est elle aussi constituée d'un correcteur Proportionnel-Intégral (PI) noté $C_{1}(s)$. La mesure vitesse moteur est déduite par dérivation numérique de la mesure position moteur. La boucle de position est constituée d'un correcteur Proportionnel $(P)$ noté $C_{2}(s)$ suivi d'un filtre passe-bas appelé filtre de consigne vitesse noté $F_{\text {fcv }}(s)$ :

$$
C_{1}(s)=K_{v} \frac{1+\tau_{\mathrm{i}} s}{\tau_{\mathrm{i}} s}, C_{2}(s)=K_{\mathrm{p}} \text { et } F_{\mathrm{fcv}}(s)=\frac{1}{1+\tau_{\mathrm{fcv}} s}
$$

Le réglage respectant le cahier des charges présenté dans la section 2.1 est obtenu pour les valeurs suivantes :

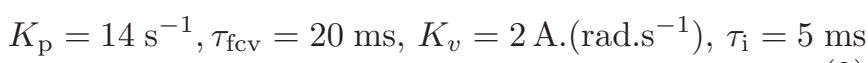

Le temps de cycle obtenu expérimentalement (Fig. 5a) sur notre trajectoire de référence est de 1,1 s. Il est important de noter que la position moteur, le courant moteur et surtout la position du bout du bras sont très oscillants. Cette commande est pour l'instant celle qui est actuellement utilisée en industrie, sa robustesse n'est donc plus à démontrer.

\section{Loi en jerk limité}

Dans cette partie, la référence en accélération limitée est remplacée par une référence en jerk limité (Fig. 6). Il a été montré dans [4] que si la valeur de jerk maximal réglée correspond au niveau vibratoire attendu, la loi à jerk limité réalise un bon compromis entre temps de mouvement et vibrations résiduelles. La première fréquence de résonance du robot manipulateur sur la trajectoire de référence est à $12,5 \mathrm{~Hz}$, la valeur maximale de jerk à appliquer est donnée par :

$$
J_{\max }=A_{\max } \cdot f_{\text {résonance }}=50 \mathrm{~m} \cdot \mathrm{s}^{-3}
$$



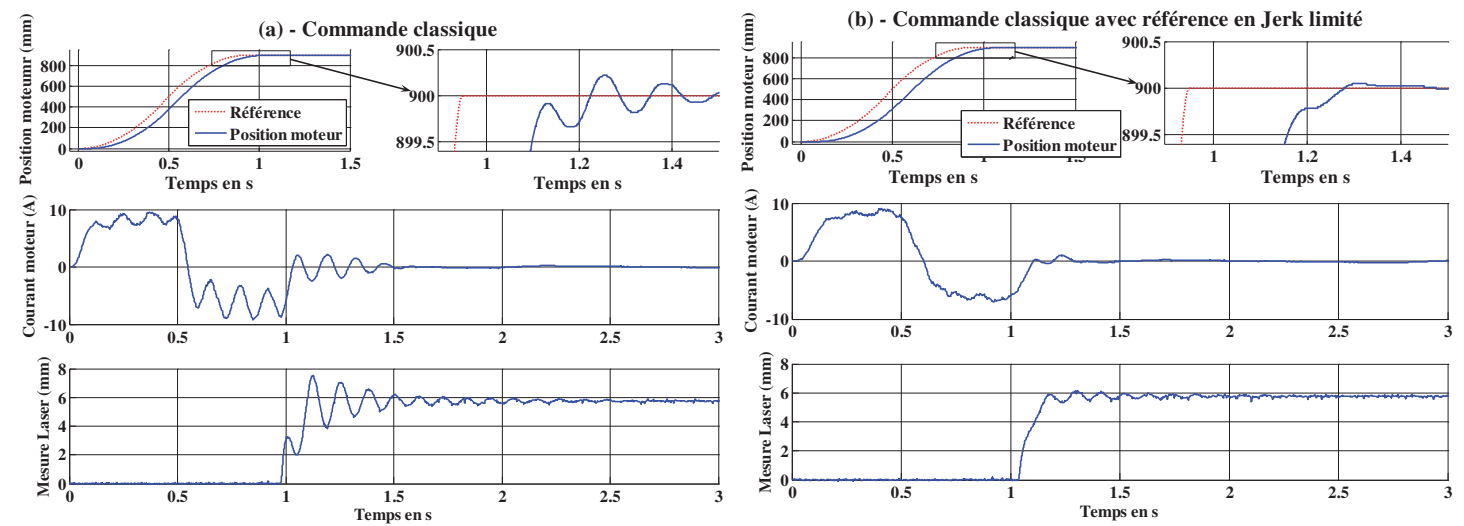

Fig. 5. Résultats expérimentaux (a) - commande classique (b) - commande classique avec référence en jerk limité.
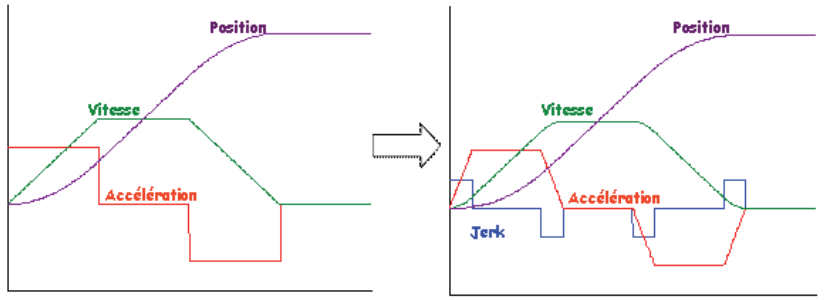

Fig. 6. Passage d'une référence en accélération limitée en jerk limité.

L'intérêt majeur est que la loi à jerk limité est disponible dans les commandes numériques du marché. Elle permet un meilleur comportement vibratoire mais cette amélioration se fait au détriment du temps de cycle. Les résultats expérimentaux le confirment (Fig. 5b), le comportement vibratoire au niveau de la charge est meilleur mais au détriment du temps de cycle (perte de 4,5\% en temps de cycle). La loi à jerk limité a un intérêt pratique immédiat :

- Le choix de la valeur maximum du jerk, qui conduit à un niveau d'oscillation désiré, dépend principalement de la position fréquentielle de la souplesse prépondérante et de l'accélération maximale de l'axe ainsi que de son gain de position et de son pourcentage d'anticipation. Les essais expérimentaux sur démonstrateur ont permis de montrer que l'influence du jerk pouvait être correctement estimée « a priori » par la formulation théorique développée dans [4]. Cette démarche permet d'éviter le recours classique aux techniques « essais et erreurs ».

- La gestion du jerk couplée à la modulation du gain de position permet d'obtenir un compromis raisonnable entre niveau des vibrations résiduelles et durée du mouvement. Des essais supplémentaires montreraient qu'il est même possible de conserver le temps de cycle originel (sans gestion du jerk) tout en minimisant les vibrations de la charge.

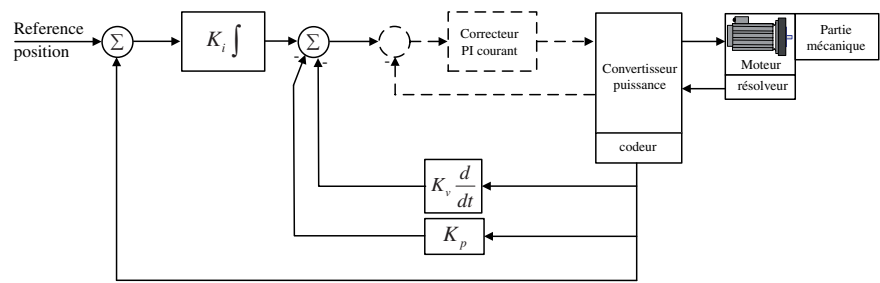

Fig. 7. Structure de la commande par retour d'état.

\section{Commande par retour d'état}

Cette commande se distingue par le fait que la contreréaction n'est pas appliquée seulement sur un écart mais sur toutes les variables régissant le comportement dynamique du système [5]. Ces variables sont appelées variables d'état. Le modèle retenu pour le réglage de cette commande est un modèle complètement rigide. Le comportement du système est donc régi par un système du premier ordre :

$$
m_{\mathrm{eq}} \ddot{x}=U-f \dot{x}
$$

où $M_{\text {éq }}$ correspond à la masse totale en mouvement, $f$ au coefficient de frottements visqueux, $x$ à la position moteur et $U$ à l'effort de poussée provenant du moteur. Les variables d'état utilisées correspondent à la vitesse et la position moteur auxquelles se rajoute l'intégrale de l'erreur de position ce qui permet d'annuler toutes les erreurs statiques venant d'une perturbation constante (un frottement sec typiquement). La structure de commande par retour d'état est présentée figure 7. L'algorithme de commande a pour expression :

$$
i_{\text {reg }}=K_{\mathrm{i}} \int\left(x_{\text {réf }}-x\right)-K_{\mathrm{v}} \dot{x}-K_{\mathrm{p}} x
$$

Il existe de nombreuses méthodes de réglage des gains $K_{\mathrm{i}}, K_{\mathrm{v}}$ et $K_{\mathrm{p}}$ (minimisation de critère, commande robuste, etc.), la méthode retenue est la méthode par placement de pôles. En effet, le même placement de pôles que celui réalisé par la commande «classique » a été retenu pour obtenir les valeurs des gains $K_{\mathrm{i}}, K_{\mathrm{v}}$ et $K_{\mathrm{p}}$. Les résultats expérimentaux (Fig. 8a) montrent que le comportement du système est beaucoup moins oscillant avec 

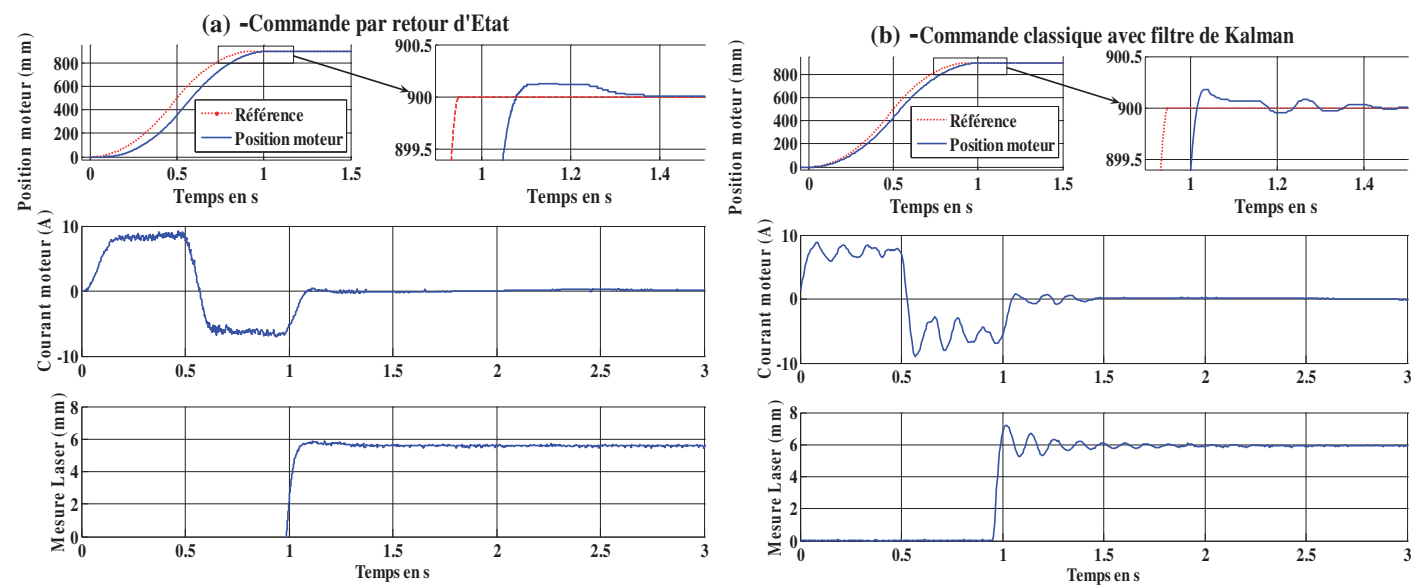

Fig. 8. Résultats expérimentaux (a) - commande par retour d'état (b) - commande classique avec filtre de Kalman.

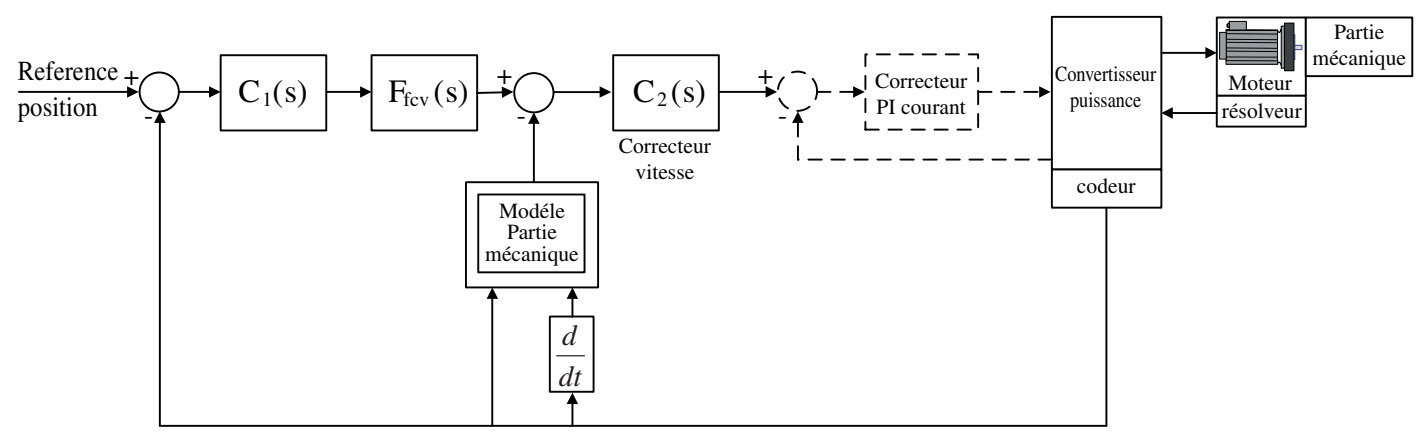

Fig. 9. Structure de la commande « classique » avec filtre de Kalman.

cette commande. Les vibrations en bout de bras ont quasiment disparu et le gain en temps de cycle est conséquent (de 1,1 s pour la commande « classique » à 1,05 s avec la commande par retour d'état soit un gain de $4,5 \%$ ). Le deuxième intérêt de cette commande est que l'algorithme est beaucoup plus simple à implanter qu'une commande par boucles en cascades. Ces résultats montrent qu'il est possible d'atteindre de meilleures performances et surtout qu'il est possible de mieux maîtriser le comportement de la boucle d'asservissement.

\section{Filtrage par filtre de Kalman}

Le bruit sur le signal de vitesse est une des causes de vibrations de notre système. Ce bruit provient essentiellement du traitement numérique réalisé sur le signal de position. Les techniques de filtrage classique (filtre passebas, filtre moyenneur, etc.) ne peuvent pas être utilisées puisque l'introduction du moindre retard dans la mesure peut déstabiliser le système et dégrader les performances de la commande. L'utilisation d'un filtre de Kalman permet d'éviter ces inconvénients. Il peut être considéré comme étant le meilleur estimateur d'état dans le cas où les signaux sont bruités [6]. Pour nos expérimentations, il a été utilisé en lien avec la commande « classique » pour remplacer la mesure vitesse (Fig. 9). Le modèle utilisé pour la synthèse du filtre de Kalman est un modèle rigide déjà présenté équation (4). Le filtre obtenu utilise le courant de référence et les mesures de vitesse et position moteur, son réglage a été effectué à partir des caractéristiques des bruits de mesures relevés expérimentalement.

La figure 10 compare le signal de vitesse mesurée au signal de vitesse estimée par filtre de Kalman. Il est important de constater que le filtre de Kalman n'introduit pas de retard dans la mesure contrairement aux techniques de filtrage classique. Les bruits de mesure étant nettement diminués, le gain de position $K_{\mathrm{p}}$ a pu être augmenté de plus de $50 \%$ tout en restant dans notre cahier des charges (Fig. 8b). Le temps de cycle est alors de $1 \mathrm{~s}$ soit un gain de $9,1 \%$ par rapport à la commande « classique» seule. Le comportement vibratoire a aussi été amélioré. On notera que le filtre de Kalman a été appliqué sur une seule architecture de commande mais aurait pu être utilisé en lien avec les autres commandes retenues dans cette étude. En effet, il ne correspond pas à une architecture de commande mais à un traitement de signal.

\section{Compensation d'un mode de transmission par retour accélérométrique}

\subsection{Modèle utilisé}

Un modèle à paramètres localisés dont la valeur de raideur dépend de la position du robot permet la 


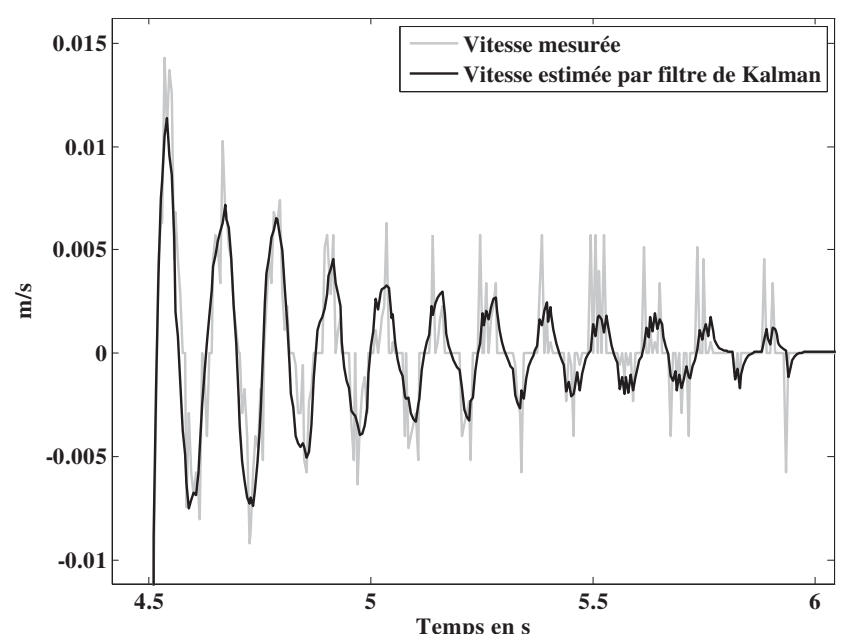

Fig. 10. Comparaison entre le signal de vitesse mesurée et le signal de vitesse filtrée (Kalman).

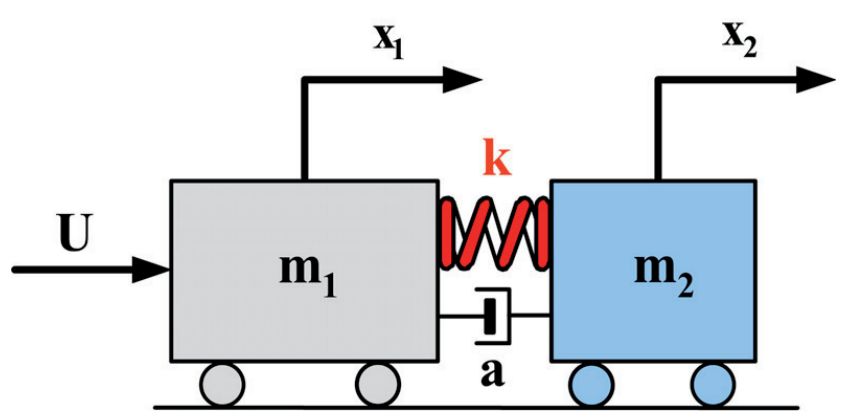

Fig. 11. Modélisation masse-ressort de l'axe $X$.

représentation des premiers modes de déformation d'un système [5-8]. Dans notre cas, des essais expérimentaux ont montré que le comportement vibratoire de l'axe $X$ est essentiellement soumis à un mode de déformation dominant. Le comportement du manipulateur peut alors être représenté par un modèle deux masses un ressort de raideur variable (Fig. 11). Les valeurs des paramètres ont été déduites d'une analyse modale expérimentale. La position $x_{1}$ de la masse $m_{1}$ correspond à la position moteur et la position $x_{2}$ de la masse $m_{2}$ à celle de la charge (extrémité du bras). L'effort $U$ correspond à l'effort provenant de l'actionneur de l'axe $X$. En utilisant le principe fondamental de la dynamique, on obtient:

$$
\begin{aligned}
m_{1} \ddot{x}_{1} & =k\left(x_{2}-x_{1}\right)+a\left(\dot{x}_{2}-\dot{x}_{1}\right)+u \\
m_{2} \ddot{x}_{2} & =k\left(x_{1}-x_{2}\right)+a\left(\dot{x}_{1}-\dot{x}_{2}\right) \\
k & =g\left(x_{2}, y_{2}, z_{2}\right)
\end{aligned}
$$

\subsection{Contrôle de la position moteur}

Supposons que l'on puisse mesurer l'accélération de la masse $m_{2}$ en plaçant un accéléromètre en extrémité de bras. On remarque que (6), (7) donne :

$$
u=m_{1} \ddot{x}_{1}+m_{2} \ddot{x}_{2}
$$

Introduisons $w$ tel que $u=w+m_{2} \ddot{x}_{2}$. L'équation (6) est alors réduite à un double intégrateur :

$$
w=m_{1} \ddot{x}_{1}
$$

L'algorithme de commande suivant peut être choisi tel que l'erreur en position $\varepsilon_{1}=x_{1}-x_{1 \text {,ref }}$ converge exponentiellement vers zéro :

$$
u=m_{2} \ddot{x}_{2}+m_{1} \ddot{x}_{1, \text { réf }}+k_{1}\left(\dot{x}_{1, \text { réf }}-\dot{x}_{1}\right)+k_{2}\left(x_{1, \text { réf }}-x_{1}\right)
$$

Les paramètres $k_{1}$ et $k_{2}$ sont de simples gains de réglage, $\ddot{x}_{1 \text {,réf }}, \dot{x}_{1 \text {,réf }}$ et $x_{1 \text {,réf }}$ correspondent aux consignes de référence présentées figure 3 et $\ddot{x}_{2}$ est la mesure d'accélération de la charge. Les résultats expérimentaux (Fig. 12a) montrent le principal intérêt de cette commande, la référence de position et la position moteur sont quasiment superposées, cependant le comportement vibratoire de la charge n'est pas totalement contrôlé.

\subsection{Contrôle des vibrations}

La méthode précédente consiste à fixer une trajectoire de référence au niveau de la position moteur. Or, le point que l'on souhaite contrôler se situe au niveau de la charge. Une autre méthode de contrôle consiste à définir une trajectoire de référence pour la position de la charge (sans vibrations bien sûr). La trajectoire de référence au niveau moteur est déduite en utilisant le principe d'inversion et en dérivant la relation (6) par rapport au temps :

$$
\begin{aligned}
a \ddot{x}_{2, \text { réf }}= & -k\left(x_{2, \text { réf }}, y_{2, \text { réf }}, z_{2, \text { réf })} \dot{x}_{1, \text { réf }}\right. \\
& -\frac{\mathrm{d} k}{\mathrm{~d} t}\left(x_{2, \text { réf }}, y_{2, \text { réf }}, z_{2, \text { réf }}\right) \\
& +a \ddot{x}_{2, \text { réf }}+m_{2} \ddot{x}_{2, \text { réf }}+k\left(x_{2, \text { réf }}, y_{2, \text { réf }, ~}, z_{2, \text { réf })} \dot{x}_{2, \text { réf }}\right. \\
& +\frac{\mathrm{d} k}{\mathrm{~d} t}\left(x_{2, \text { réf }}, y_{2, \text { réf }}, z_{2, \text { réf }}\right)
\end{aligned}
$$

Cette inversion peut se faire hors-ligne et les problèmes de causalité ne sont pas à prendre en compte dans ce calcul puisque les références que l'on se fixe sont entièrement connues (leur évolution est fixée). Une fois la trajectoire de référence moteur obtenue, elle est envoyée comme commande dans l'algorithme (11). Pour résumer, la position moteur suit une référence qui impose une trajectoire fixée à la charge. Les résultats obtenus (Fig. 12b) montrent que la position de la charge est cette fois-ci parfaitement contrôlée (les vibrations résiduelles ont totalement disparu). Le contrôle des vibrations de la charge se fait directement ressentir sur la forme du courant moteur sur lequel des discontinuités apparaissent.

Remarque : L'équation (12) montre que le jerk doit être défini pour réussir à inverser le modèle. Pour nos expérimentations, la trajectoire de référence utilisée est la même que celle utilisée dans la section 4 . 

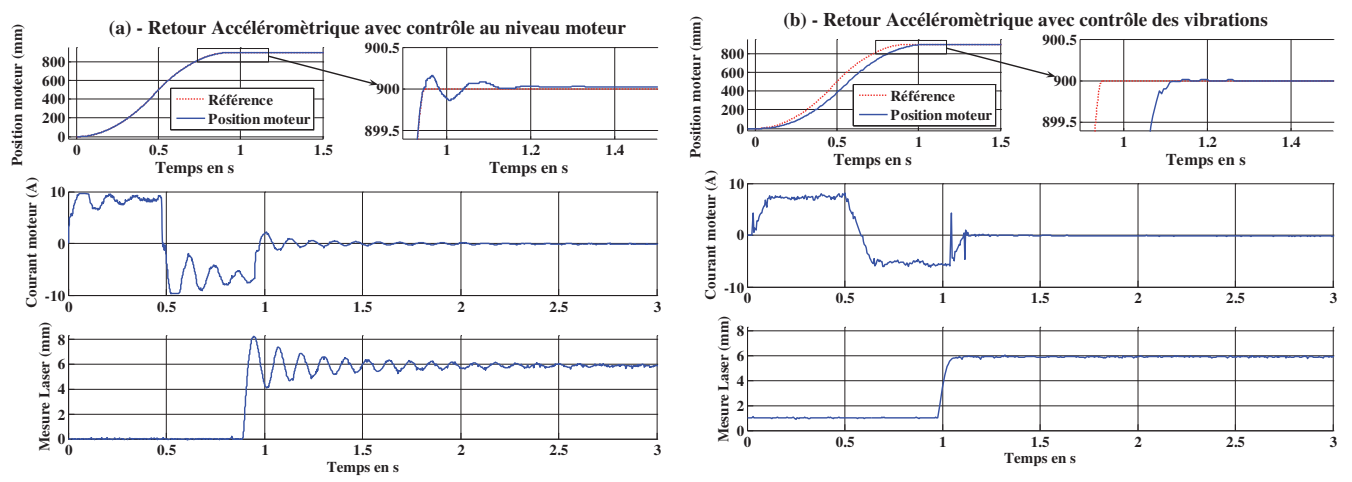

Fig. 12. Résultats expérimentaux (a) - commande par retour accélérométrique (contrôle au niveau moteur) (b) - commande par retour accélérométrique (contrôle des vibrations).

Tableau 1. Comparaison des performances des différents types de commandes retenues.

\begin{tabular}{|c|c|c|}
\hline Architecture de commande & Temps de cycle & $\begin{array}{c}\text { Comportement } \\
\text { vibratoire }\end{array}$ \\
\hline Commande « classique » & Moyen & Faible \\
\hline Commande « classique $»$ avec & & \\
\hline référence en jerk limité & Faible & Excellent \\
\hline Commande par retour d'état & Bon & Bon \\
\hline $\begin{array}{l}\text { Commande « classique » avec filtre de } \\
\text { Kalman }\end{array}$ & Bon & Moyen \\
\hline $\begin{array}{l}\text { Commande par retour } \\
\text { accélérométrique (contrôle moteur) }\end{array}$ & Excellent & Faible \\
\hline $\begin{array}{l}\text { Commande par retour } \\
\text { accélérométrique (contrôle des } \\
\text { vibrations) }\end{array}$ & Bon & Excellent \\
\hline
\end{tabular}

\section{Conclusion}

Cet article a proposé une analyse comparative des performances de différentes structures de commande. Les résultats présentés sont spécifiques à une application particulière, celle d'un robot cartésien. Les critères de comparaison sont ici la durée effective du mouvement ainsi que le niveau d'oscillations résiduelles sur la charge. Les conclusions auxquelles nous sommes parvenus sont résumées dans le tableau 1.

La structure de commande par retour accélérométrique permet une modulation facile du compromis temps/vibration. Dans le cas où l'ajout d'un capteur supplémentaire n'est pas envisageable, une action sur la loi de mouvement (jerk) permet d'une manière très simple de réaliser un compromis satisfaisant. La commande par retour d'état, bien que proche de la structure classique, conduit à de meilleures performances en termes de réduction de vibrations.

On notera que la recherche d'un optimum pour le compromis temps/vibrations résiduelles se retrouve pour la plupart des systèmes de positionnement réalisant des mouvements de type point à point. On notera également que les structures de commandes présentées ne nécessitent pas une algorithmique importante, donc sont toutes potentiellement implantables dans les systèmes de commande modernes.

\section{Références}

[1] D. Brun-Picard, F. Malburet, Contrôle actif des vibrations : ce qui marche et ce qui ne marche pas, Deuxième assises « Machines et Usinage à Grande Vitesse », ENSAM CER de Lille, 13-14 mars 2002

[2] G. Zhang, J. Furusho, Speed control of two-inertia system by PI/PID control, IEEE transactions on industrial electronics, 2000, p. 47

[3] G. Ellis, R.D. Lorenz, Comparison of motion control loops for industrial applications, IEEE industry applications conference, 1999, p. 4

[4] R. Béarée, P.-J. Barre, E. Dumetz, F. Ghestem, H. David, Influence du jerk sur le comportement dynamique d'un robot cartésien 3 axes, Troisième assises « Machines et Usinage à Grande Vitesse », IFMA de Clermont-Ferrand, 10-11 mars 2004

[5] P. Borne, G. Dauphin-Tanguy, J.P. Richard, F. Rotella, I. Zambettakis, Modélisation et identification des processus, Tome 1, Technip, 1992

[6] R.E. Kalman, A new approach to linear filtering and prediction problems, Trans. ASME J. Basic Engineering 82 (1960) 34-45

[7] P.J. Barre, J.P. Hautier, J. Charley, The use of modal analysis to improve the axis control, Fourth International Congress on Sound and Vibration, St. Petersburg, Russia, 1996, pp. $1531-1538$

[8] E. Dumetz, J.-Y. Dieulot, P.-J. Barre, F. Colas, T. Delplace, Control of an industrial robot using acceleration feedback, J. Intelligent Robotic Systems 46 (2006) 111-128 